\title{
Characterization of the relationship between semantic and structural language features in psychiatric diagnosis
}

\author{
N.B.Mota \\ Brain Institute \\ UFRN Natal \\ Brazil \\ F.Carrillo \\ Department of \\ Computation \\ UBA Buenos \\ Aires \\ Argentina
}

\author{
D.F.Slezak \\ Department of \\ Computation \\ UBA Buenos \\ Aires \\ Argentina
}

\author{
M.Copelli \\ Physics \\ Department \\ UFPE Recife \\ Brazil
}

\author{
S.Ribeiro \\ Brain Institute \\ UFRN Natal \\ Brazil
}

\begin{abstract}
Psychiatry describes speech symptoms that are indicative of disorganized thought, but measuring them is not easy. With natural language processing tools, it is possible to quantify psychiatric symptoms. Graph representations of word trajectories and semantic incoherence have independently been shown to predict the Schizophrenia diagnosis. Both analyses assess thought organization through speech, but the relationship between them is unknown. To fill this gap, here we characterize the relationship between structural and semantic features of free verbal reports from 60 patients and matched controls. Graph connectedness is inversely correlated to semantic incoherence and both explain 54\% of negative symptoms variance.
\end{abstract}

\section{INTRODUCTION}

For over a century, psychiatry has described speech symptoms perceived by the specialist as indicative of disorganized thought [1]. The descriptions used by psychiatrists to identify thought disorders focus on aberrant trajectories in word sequences used by patients to report their memories. While mild severity is described as, for instance, 'loss of associations', higher severity may be described as 'derailment', reaching in extreme cases an apparent randomness described clinically as 'word salad'. However, even for very well trained psychiatrists, the aberrant thought organization identified through language is hard to measure with precision and without subjective biases. The development of natural language processing tools now enable us to quantify aberrant word trajectories analyzing structural [2-4] as well semantic features on patient reports $[5,6]$.
Semantic incoherence between consecutive sentences is increased in verbal reports of schizophrenic patients [6], a feature that has been shown to predict Schizophrenia even during the prodromal phase, nearly 3 years before the first psychotic break [5]. On the other hand, the representation of word trajectories as directed graphs has revealed that subjects with chronic psychosis speak with significantly less connectedness between words than healthy subjects, and this allows for the automated diagnosis of Schizophrenia [4, 7]. Importantly, connectedness attributes were negatively correlated with the severity of negative symptoms measured during standard psychiatric evaluations [3]. The set of symptoms known as negative symptoms is associated with the Schizophrenia diagnosis, poor prognosis and major impacts in social behavior [8]. Both structural and semantic measures assess thought organization through word trajectories, but the relationship between structure (word graph connectedness) and semantics (language incoherence) is yet to be mapped. Are these measures redundant or complementary? Could the combination of structural and semantic analyses improve the quantification of negative symptoms?

To address these questions, we aimed in the present study to characterize the relationship between structural and semantic features of verbal reports from patients with and without psychotic symptoms (same dataset as [3]). The study also assessed whether the combination of structural and semantic features explains the severity of negative symptoms better than the same features separately.

\section{METHODS}

A total of 40 psychotic patients (20 with Schizophrenia diagnosis and 20 with 
Bipolar Disorder diagnosis), and 20 control subjects without psychotic symptoms were interviewed during psychiatric assessment at public clinical services in Natal, Brazil. Participants and legal guardians provided written informed consent. The study was approved by the UFRN Research Ethics Committee (permit \#102/06-98244). In order to establish the diagnosis according to DSM IV, SCID was applied [9]. A psychometric scale PANSS [10] was also applied to measure psychiatric symptoms according to psychiatric evaluation. For the analysis we used the total value of the PANSS negative subscale. Next the participants were requested to report a dream, and this report was audio recorded and transcribed.

To assess structural features, each report was represented as a graph in which each word corresponded to a node, and the temporal sequence of two consecutive words corresponded to an edge. In order to control for verbosity differences, a graph was performed for each set of 30 consecutive words, with one word of difference to perform the next graph. Three connectedness graph attributes were assessed for each graph: The amount of edges (E), the amount of nodes in the largest connected component (LCC), and the amount of nodes in the largest strongly connected component (LSC). After calculating graph attributes for all 30-word graphs, the average of each attribute was calculated and considered for the analysis. Graphs analysis was performed using the software SpeechGraphs [3].

To assess semantic features, we calculated the median semantic distances between consecutive sentences using latent semantic analysis (LSA), a measure known as first order incoherence, also predictive of the Schizophrenia diagnosis [5]. To control for verbosity differences, semantic distances were normalized by the largest sentence [5]. All the statistical analysis was performed with Matlab software.

\section{RESULTS}

We found significant differences between the groups compared (Schizophrenia, Bipolar and Control groups), both for structural connectedness and for semantic incoherence (Figure 1 and Table 1). The Schizophrenia group produced less connected graphs (fewer Edges, smaller LCC and LSC) compared to the Control group, and also compared to the Bipolar group (fewer Edges and smaller LSC) (Figure 1 and Table 1). The Bipolar group also produced less connected graphs in comparison with the Control group (Figure 1 and Table 1).
In addition, the Schizophrenia group produced reports that were less semantically coherent than those of the Control group (Figure 1 and Table $1)$.

When we analyzed all subjects together, Edges, LCC and LSC were negatively correlated with median incoherence (Figure 2A). However, the relationship of semantic incoherence with Edges, LCC or LSC explained only a small portion of the data variance $(14 \%$ of the semantic incoherence variance explained by Edges, $8 \%$ explained by LCC and $15 \%$ explained by LSC, as estimated by Pearson's $\mathrm{R}^{2}$ ). Moreover, these correlations tend to persist only for the Schizophrenia group after sorting the participants according to their groups, (for the Schizophrenia group: Semantic Incoherence versus Edges $p=0.0855$, versus $L C C p=0.1056$, and versus LSC $\mathrm{p}=0.0813$ ) (Figure 2B).

Since the semantic and structural features seem to share some information but without much redundancy, we combined the three connectedness attributes with the median semantic incoherence to assess the multilinear correlation of these features with the severity of negative symptoms measured by the PANSS negative subscale. The combination of both strategies was able to explain $54 \%$ of the variance in the severity of negative symptom $\left(\mathrm{R}^{2}=0.54, \mathrm{p}<0.0001\right)$ (Figure 3).
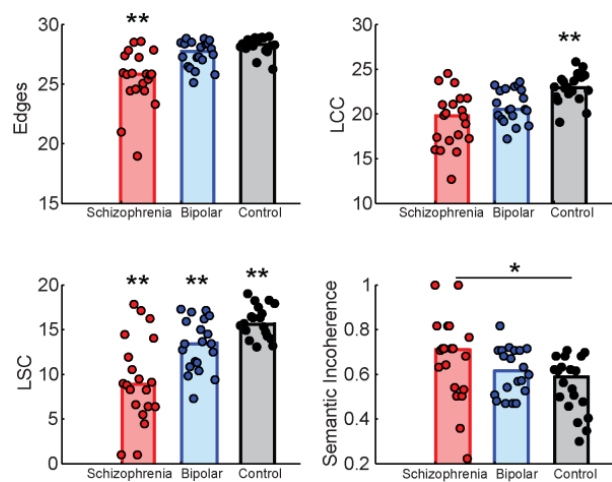

Fig 1. Dispersion plot of graph connectedness attributes and median semantic incoherence. $*$ means a group differs from another and $* *$ means a group differs from the others 2 groups

Table I: P value of Wilcoxon Ranksum test between groups. Significant results in boldface (Bonferroni corrected for 3 comparisons $-\mathrm{SxB}, \mathrm{SxC}$ and $\mathrm{BxC}-\alpha=0.0167)$.

\begin{tabular}{|c|cccc|}
\hline Ranksum & E & LCC & LSC & Incoherence \\
\hline S x B & $\mathbf{0 . 0 0 1 3}$ & 0.0909 & $\mathbf{0 . 0 0 5 1}$ & 0.1288 \\
S x C & $\mathbf{0 . 0 0 0 0}$ & $\mathbf{0 . 0 0 0 2}$ & $\mathbf{0 . 0 0 0 1}$ & $\mathbf{0 . 0 0 7 9}$ \\
B x C & 0.0275 & $\mathbf{0 . 0 0 3 1}$ & $\mathbf{0 . 0 0 6 6}$ & 0.1069 \\
\hline
\end{tabular}



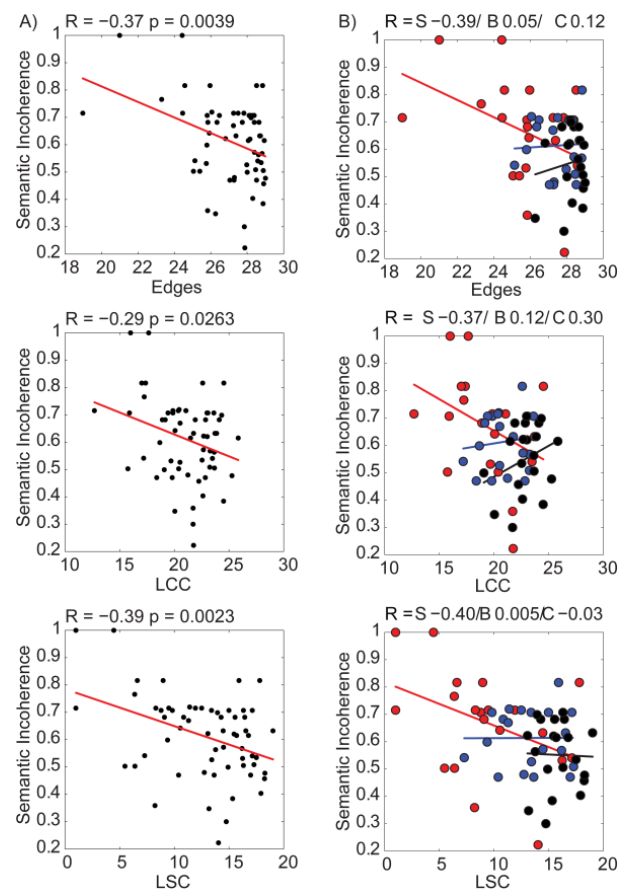

Fig 2. A) Pearson correlations between graph connectedness attributes and semantic incoherence. B) Pearson correlations between graph connectedness attributes and semantic incoherence for the Schizophrenia group ( $\mathrm{S}$ in red), Bipolar group ( $\mathrm{B}$ in blue) and Control group ( $\mathrm{C}$ in black).

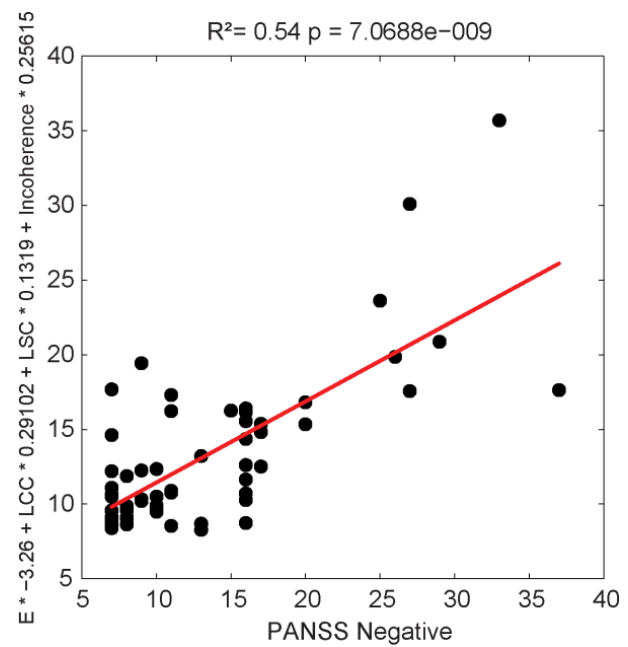

Fig 3. Multilinear correlation between structural and semantic measures and PANSS negative subscale. In y axis the coefficients founded for each attribute is described.

\section{DISCUSSION}

The results point to an inverse relationship between graph connectedness $(\mathrm{E}$, LCC and LSC) and semantic incoherence (median distance between consecutive sentences). This means that the less connected the verbal report is, the more semantically incoherent it is. Both the structural and the semantic approaches study the same object (memory reports) in order to quantify similar phenomenology (thought disorganization), but graph connectedness was able to explain only a small percentage of the variance in semantic incoherence when all subjects were considered, which indicates that these measurements are largely complementary. When we studied the correlations inside each group no significant correlations were found, and only in the Schizophrenia group - the main psychiatric pathology associated with thought disorganization - the effect tended to keep the same direction.

One limitation of the study is that the results are impacted by the small number of subjects in each group, and thus future work is necessary to better characterize the relationship between structure and semantics in a larger sample.

Notwithstanding, the combination of structural and semantic features explained more than half of the variance of negative symptoms severity. The results show that the combination of both strategies to quantitatively assess negative symptoms is an important direction that should be pursued in a larger sample.

\section{REFERENCES}

[1] H. I. Kaplan and B. J. Sadock, Kaplan \& Sadock's Comprehensive Textbook of Psychiatry: Wolters Kluwer, Lippincott Williams \& Wilkins, 2009.

[2] N. B. Mota, M. Copelli, and S. Ribeiro, "Computational Tracking of Mental Health in Youth: Latin American Contributions to a Low-Cost and Effective Solution for Early Psychiatric Diagnosis," New Dir Child Adolesc Dev, vol. 2016, pp. 59-69, Jun 2016.

[3] N. B. Mota, R. Furtado, P. P. Maia, M. Copelli, and S. Ribeiro, "Graph analysis of dream reports is especially Ribeiro, "Graph analysis of dream reports is especially
informative about psychosis," Scientific Reports, vol. 4, p. 3691,2014

[4] N. B. Mota, N. A. Vasconcelos, N. Lemos, A. C. Pieretti, O. Kinouchi, G. A. Cecchi, et al., "Speech graphs provide a quantitative measure of thought disorder in psychosis," PLoS One, vol. 7, p. e34928, 2012.

[5] G. Bedi, F. Carrillo, G. A. Cecchi, D. F. Slezak, M. Sigman, N. B. Mota, et al., "Automated analysis of free speech predicts psychosis onset in high-risk youths," npj Schizophrenia, 2015.

[6] B. Elvevåg, P. W. Foltz, D. R. Weinberger, and T. E. Beld "Qu W. Foltz, D. Weinberer, and T. E automated methodology and novel application to schizophrenia," Schizophrenia Research, vol. 93, pp. 304-

N. B. Mota, R. Furtado, P. P. Maia, M. Copelli, and S. Ribeiro, "Graph analysis of dream reports is especially informative about psychosis," Sci Rep, vol. 4, p. 3691, 2014. S. F. Austin, O. Mors, E. Budtz-Jorgensen, R. G. Secher, C. R. Hjorthoj, M. Bertelsen, et al., "Long-term trajectories of positive and negative symptoms in first episode psychosis: A 10year follow-up study in the OPUS cohort," Schizophr Res, vol. 168, pp. 84-91, Oct 2015.

[9] M. H. First, R. L. Spitzer, M. Gibbon, and J. Williams, Structured Clinical Interview for DSM-IV Axis I Disorders Research Version, Patient Edition (SCID-I/P). New York: Rem Biometrics Research, New York State Psychiatric Institute,

[10] S. R. Kay, A. Fiszbein, and L. A. Opler, "The positive and negative syndrome scale (PANSS) for schizophrenia," Schizophr Bull, vol. 13, pp. 261-76, 1987. 